Voix et Images

volxetimages

\title{
À l'origine de toutes les origines
}

\section{Lucie Robert}

Volume 31, numéro 3 (93), printemps 2006

Élise Turcotte

URI : https://id.erudit.org/iderudit/013246ar

DOI : https://doi.org/10.7202/013246ar

Aller au sommaire du numéro

\section{Éditeur(s)}

Université du Québec à Montréal

\section{ISSN}

0318-9201 (imprimé)

1705-933X (numérique)

Découvrir la revue

\section{Citer cet article}

Robert, L. (2006). À l'origine de toutes les origines. Voix et Images, 31(3), 146-155. https://doi.org/10.7202/013246ar d'utilisation que vous pouvez consulter en ligne.

https://apropos.erudit.org/fr/usagers/politique-dutilisation/ 


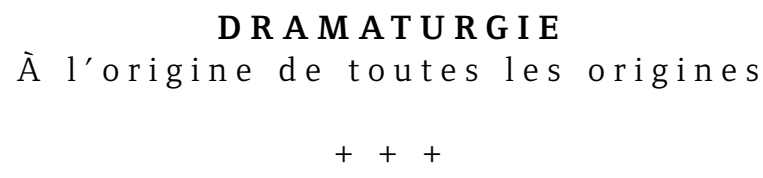

LUCIE ROBERT

Université du Québec à Montréal

«L'humanité, qui découvre sans cesse du sens, ne peut toujours inventer de nouvelles formes, et il lui faut parfois investir de sens nouveaux des formes anciennes ${ }^{1}$ ", écrivait Gérard Genette. Inscrire des sens nouveaux dans des formes anciennes est peut-être l'enjeu qui réunit les textes dont il sera question ici. Car, au thêâtre, et sans doute plus que dans n'importe quel autre genre de l'écriture littéraire, la tradition du palimpseste est bien présente. Depuis deux millénaires, les dramaturges réinventent les mêmes fables, réinterprètent les mêmes événements, reconstruisent les mêmes schémas. Nombreux sont les auteurs qui reviennent sur des textes antérieurs qu'ils relisent, corrigent, récrivent, en plus long ou en plus court. Et que dire du jeu de l'acteur ou de la mise en scène qui sont fondés, dans leur principe même, sur la lecture ou la relecture de textes déjà-là ? Ce qui importe au thêâtre, en effet, n'est peut-être pas tant l'originalité de l'histoire racontée que la manière dont on utilise cette histoire pour représenter l'être humain, qu'il soit confronté au monde extérieur ou à son monde intérieur, et pour repenser le sens de son histoire à lui, voire le sens de l'Histoire elle-même.

À travers ces multiples redites, apparaît la volonté de remonter aux sources de l'Histoire, pour rappeler à la mémoire des vivants que notre civilisation s'est construite sur un fond de violence. "À l'origine de toutes les origines", est ainsi le titre du prologue de Tristan et Iseult, la pièce de Pierre-Yves Lemieux dont il sera tout de suite question. Comme s'il fallait en arriver à laisser entendre que, malgré la variation de ses formes, et tant que l'humanité sera ce qu'elle est, le monde ne pourra qu'être à son image et à sa ressemblance, c'est-à-dire qu'il ne sera jamais que la répétition de lui-même, et qu'il faut peut-être envisager l'hypothèse selon laquelle l'Histoire n'aura jamais été qu'une succession de palimpestes. Et s'il est un second enjeu qui réunit les textes qui suivent, c'est bien de déployer cette éthique de la répétition dans une esthétique qui repose sur le fragment. Plutôt qu'une action unique qui se déploierait dans le temps, ces textes présentent le plus souvent une

1 Gérard Genette, Palimpsestes. La littérature au second degré, Paris, Éditions du Seuil, coll. "Points essais », 1982, p. 558 . 
succession de tableaux ou de micro-récits dont l'unité résulte de la présence d'un personnage - le Poète, la Mémoire, la Didascalienne, l'Ange annonciateur, voire Gisèle Schmidt -, qui lui donne un cadre, une logique, une cohérence. La narrativité du thêâtre contemporain est un phénomène déjà bien établi ${ }^{2}$, mais ses formes n'en finissent plus de bouger. En conséquence, on ne s'étonnera guère de trouver un certain nombre d'appellations, "roman-dit», "conte» ou "nouvelles-thêâtre», qui accentuent le brouillage entre les genres et nous invitent à lire encore autrement.

Pièce en cinq parties, avec prologue et épilogue, Tristan et Yseult ${ }^{3}$ de Pierre-Yves Lemieux s'ouvre sur le discours du poète Ymir: «Mon conte se déroulera tout juste après le passage des divinités [...]. Ce chant que je recrée inlassablement depuis l'origine de toutes les origines,/bien d'autres se l'ont approprié,/au fil des temps,/ sous diverses formes,/sous divers noms. " (9) Il ne sera toutefois pas question pour l'auteur de reprendre l'histoire connue "sous d'autres noms», mais au contraire de revenir à une version donnée comme authentique sinon originale, plus proche de la tradition celtique, dont l'auteur reprend les motifs tout en les développant. Parti à la recherche de "celle à qui appartient ce cheveu d'or" (32), que le roi Marc'h entend épouser, Tristan doit d'abord mener deux combats : tuer le monstre, le gardien de l'île verte, puis tuer la bête, qui terrorise les habitants. Deux fois vainqueur, il en ressort chaque fois mourant, la première sous l'effet d'un poison, la seconde, sous l'effet d'un maléfice. Sauvé par la Mère, puis par la fille (Yseult), Tristan est néanmoins victime d'une prophétie où Yseult a été promise à celui qui tuera la bête. Celle-ci le sait, qui envoûte Tristan et vit un grand amour pendant les quelques instants qu'il lui reste, avant d'être livrée à Marc'h afin de «redonner la paix aux îles» (86), enfin réunies. Marc'h sent pourtant l'émotion qui lie le destin de Tristan et Yseult, dont l'amour survit à la fin de l'envoûtement. Jaloux, il les condamne, puis renonce et se noie dans la rivière. Quand Tristan rappelle Yseult vers lui, il croit d'abord qu'elle ne vient pas et se suicide. Elle était là pourtant et, de désespoir, meurt aussi. Si l'histoire s'arrête, c'est que "[l]eur passion prend place au sein du monde vivant» et que «[a]u-delà est une histoire que nul n'a le droit de conter» (160). La relecture que propose Pierre-Yves Lemieux prend ainsi la forme d'une tragédie aux accents shakespeariens, par le recours au merveilleux que suggérait la tradition orale, par l'inscription des enjeux politiques qu'est la réunion des deux îles, mais également par la fin qui n'est pas sans rappeler celle de Roméo et Juliette. Dès lors, la structure de l'action répond aux exigences de la tragédie classique où, explique Ymir, "[1]a grandeur des hommes ne saurait se chanter/sans grands périls et douleurs à franchir» (138). La pièce est versifiée dans un langage qui confère à la tragédie des

2 On lira sur ce point Chantal Hébert et Irène Perelli-Contos (dir.), Le théâtre et ses nouvelles dynamiques narratives, Québec, Les Presses de l'Université Laval, 2004. 3 Pierre-Yves Lemieux, Tristan et Yseult, Montréal, Lanctôt éditeur, 2004, 160 p. 
accents poétiques que vient accentuer le Poète, sorte de Coryphée moderne. C'est à lui que revient la fonction de donner un sens aux événements représentés, sens qui échappe aux personnages. Ymir refuse de concéder la primauté aux enjeux politiques: «Toutes les puissances du monde ne sont rien si leur monde n'est plus.» (159)

Avec Les Atrides. D'après Eschyle, Sophocle, Euripide, Sénèque et Jean Giraudoux ${ }^{4}$, et comme Pierre-Yves Lemieux, Denyse Noreau reprend une histoire que le thêâtre «recrée inlassablement depuis l'origine de toutes les origines». La pièce commence par un prologue où Mnémosyne, déesse de la mémoire et mère des Muses annonce: «[J]e suis celle que les souvenirs assaillent.» (13) La figure du Poète est ainsi remplacée par la Mémoire elle-même, convoquée non pas tant pour rappeler la tragique histoire des Atrides que pour répondre à la requête d'Oreste, qui tient à laisser à ses descendants «l'assurance que le temps de tout ce sang gâché est bien terminé» (20). Denyse Noreau reprend donc l'histoire du début, c'est-à-dire depuis Tantale, «le premier a osé défier les dieux» (25). Suivront Pélops, fils de Tantale, puis ses fils à lui, Atrée et Thyeste, qui «allaient pousser l'horreur si loin qu'après eux, les humains ne pourraient plus jamais prétendre dépasser un crime aussi perfide» (34), et enfin le couple formé par Agamemnon et Clytemnestre. À travers le récit, d'abord pris en charge par un vieux serviteur que Mnémosyne relaie au besoin, Oreste se rappelle ces événements douloureux, qui se révèlent finalement trop lourds à porter. Mnémosyne insiste cependant: «Je t’ai prévenu, la Mémoire ne peut rien oublier.» (52) Revivent alors la guerre de Troie, le sacrifice d'Iphigénie, puis les assassinats d'Agamemnon, d'Égisthe et de Clytemnestre. À ce moment seulement, le récit s'arrête, car l'histoire des Atrides s'est interrompue. Clytemnestre l'avait prédit à Oreste: "Ta sœur et toi resterez toujours les enfants d'Agamemnon. Vous ne deviendrez jamais autre chose. [...] Vous serez figés dans l'enfance, il n'y aura plus rien devant vous.»(80) Figé dans ce présent éternel, Oreste comprend que les Atrides n'ont toujours vécu que de "grandeurs illusoires» (83), dans un esprit de vengeance immédiat où les intérêts supérieurs (de la Cité ou des Dieux) n'ont jamais vraiment eu de place: «[J]e ne veux pas mourir en te ressemblant» (83), dit-il à son père.

Créé au Théâtre de la Cité universitaire de l'Université Laval, les 6, 7 et 8 décembre 2001, dans une mise en scène de Puma Freytag, Les Atrides est un texte conçu pour le théâtre de marionnettes, ce qui a pour effet de réduire les exigences de la scène en matière d'action et, surtout, d'atténuer l'horreur de ces mille et un événements racontés en l'espace d'une seule nuit. Le texte poursuit visiblement une visée didactique, d'une part, par la conception d'une sorte de condensé de l'histoire familiale des Atrides et, d'autre part, par la réflexion qu'elle propose sur la violence,

4 Denyse Moreau, Les Atrides. D'après Eschyle, Sophocle, Euripide, Sénèque et Jean Giraudoux, adaptation et composition du texte par Denyse Moreau, Québec, Éditions Va bene, 2004, 84 p. 
la guerre et leurs effets destructeurs. Mnémosyne le réaffirme : «La Mémoire ne sait dire que le passé. Mais en lui est tout un enseignement.» (20) Ainsi la pièce peut être vue aussi comme la volonté d'introduire un peu de sens commun dans un des grands récits fondateurs de la civilisation occidentale, et de rappeler ce qu'il est réellement: une histoire de bas intérêts et de violence incontrôlée: «[J]e te dis qu'il faut que les hommes se souviennent du sang versé, qu'ils entendent les cris et les pleurs qui montent des champs de bataille à la fin des combats. L'horreur, Calchas, a toujours la même odeur.» (64)

Des Atrides aux apatrides, y a-t-il une si grande distance? L'assonance est évidemment séduisante. Et peut-être, en effet, peut-on déployer l'hypothèse d'une sorte de proximité sémantique qui ramènerait à l'avant-plan la réflexion sur la guerre et sur les conséquences de la violence. Car chez les apatrides, violemment dépouillés de leur espace et de leur temps, la mémoire, seule, maintient la cohésion de la communauté. L'espoir aussi, sans doute un peu, dans la mesure où il permet une sorte de projection dans l'avenir. Le récit occuperait alors une fonction essentielle puisqu'il assure la continuité. Pourtant, la pièce que Marilyn Perrault a intitulée Les apatrides ${ }^{5}$ ne renvoie pas exactement au propos attendu. Créée à l'Espace libre le $1^{\text {er }}$ octobre 2003, dans une mise en scène de Marc Dumesnil, elle s'inscrit plutôt dans un paysage résolument urbain et policé, dont elle révèle le côté sombre. Le personnage principal est une enfant abandonnée, dont le credo (le credo des bâtards) est: "Je viens de nulle part, je suis partout pis je m'en vas ailleurs.» (8) Abandon, errance sont des motifs qui laissent entrevoir, au premier abord, une quête dont la nature est plus individuelle que collective. Pourtant, certaines idées, certaines images tendent à conférer à la démarche de l'enfant un caractère collectif. Ainsi, elle parle huit huitièmes de langue «et pas une seule au compl...» (11) puis elle affirme $n^{\prime}$ «avoir le besoin de personne. Moi, je veux être autodidacte de ma vie. Moi, je veux être un pays autosuffisant» (25).

Au fil du temps, l'enfant, qui n'a pas de nom - elle est simplement Elle -, enfourche sa bicyclette et part à la rencontre d'autres personnages qui, comme elle, sont sans attaches. Elle croise d'abord la Dehors Woman, qui lui apprend quelques dures réalités de la vie d'une femme: «Je la détestais pour la réalité qu'elle m'avait fessée dans face.» (21) Puis elle fait la connaissance du clochard Clock, qui lui donne une chaîne en or pour réparer sa bicyclette, tout ce qui lui restait de son fils à lui. Ayant abandonné la première à sa triste vie, elle emmène le second avec elle. Elle se résout à faire de même pour un troisième personnage, désigné lui aussi par un pronom (Il) qui s'accroche au vélo et le fait tomber: «Qu'est-ce que vous avez tous à m'aimer aujourd'hui ?» (34) Incapable de pédaler pour tout le monde cependant, elle les abandonne peu après et poursuit son périple: «Je roule à sept mille lieues

5 Marilyn Perrault, Les apatrides, Montréal, Dramaturges éditeurs, 2005, 65 p. 
au-dessus des nuages et je me fais mon cinéma, celui de ma vie» (45), écrit-elle. Après avoir visité la Nipponie, puis le métro, Elle revient, prête à aimer Il et à fonder une famille. Mais c'est pour découvrir que Il est son frère et que l'amour leur est interdit. Peu importe: "L'amour rend aveugle, qu'y disent, Il. Ben! si y est assez aveugle, y verra pas qu'on est frère et sœur.» (62) Au théâtre, toutefois, nul ne peut se soustraire à la tragédie annoncée, de sorte que la situation se détraque : «[J]'ai, de façon grotesque, raté mon entrée dans la vie de femme adulte, hein?» (64) Il lui faut donc repartir. Trajectoire d'une enfant abandonnée, à l'identité incertaine et fluctuante, Les apatrides, première pièce de l'auteure, évite toute conclusion. Contrairement à l'errance que connaissent Il et Clock, le mode du voyage initiatique, qui n'est pas sans rappeler, bien qu'à distance, celui d'Ulysse, engage à poursuivre la quête plutôt qu'à renoncer.

C'est la guerre, en ce qu'elle a de plus conventionnel, qui est à la source de ces autres apatrides que sont ceux de Daniel Danis, héritiers du désastre, qui partagent néanmoins avec les personnages de Marilyn Perrault la quête d'une rive sur laquelle débarquer. Troisième volet de La trilogie des souliers, qui débutait avec Celle-là et Le chant du Dire-Dire, la plus récente pièce de Danis porte, en guise de titre, une seule voyelle, $e^{6}$. D'abord diffusée en version radiophonique sur France-Culture le 4 juillet 2004, dans une réalisation de Blandine Masson, la pièce a été créée au Théâtre National de la Colline, à Paris, le 26 janvier 2005, dans une mise en scène d'Alain Françon, puis jouée en tournée en France, au Canada et finalement à Montréal, au Festival de Théâtre des Amériques, en juin 2005.

La pièce se présente comme une fresque composée de micro-récits diversement intitulés «tableau», "tableautin», «miniature», mais aussi, selon l'humeur de l'auteur, qui joue sur la polysémie du mot, «tableau noir de craie», «camera oscura» ou "poudre de fusain et cire sur papier vélin ». Des tableaux proprement dits, il y en aura vingt-cinq, numérotés de A à Z. Vingt-cinq, car manque bien sûr le tableau E. Il faut donc voir dans cette voyelle, à la fois une sorte d'énigme à résoudre et le principe unificateur du propos. Le "e e apparaît là où on l'attend le moins - dans l'empreinte de la semelle qui identifiera plus tard un assassin, par exemple -, et elle n'est jamais là où on la cherche - elle manque à la pièce de tissu où l'on a brodé les mots Le corps de mon Mond. Or, on le sait, le «e», dans le système de la langue, possède deux caractéristiques qui la singularisent: d'une part, il s'agit de la seule lettre sexuée de l'alphabet, où elle désigne le féminin; d'autre part, il s'agit d'une lettre muette qui, placée à la fin d'une syllabe sonore ou d'un adjectif, ne s'entend pas. Silencieuse, elle n'en désigne pas moins, peut-être plus encore que lorsqu'elle se fait entendre dans toute sa sonorité, comme il arrive à la femme en feu qui crie «e...e...e...e...e...e»(60).

6 Daniel Danis, e. Roman-dit, Montréal, Leméac, coll. «Théâtre», 2005, 128 p. 
La pièce est sous-titrée Roman-dit. Il y a là un genre original, qui désigne le caractère narratif du discours unissant l'ensemble des tableaux. Jean-Pierre Sarrazac remarque justement que la dramaturgie moderne se déploie surtout dans les formes brèves et que les pièces longues (si l'on peut appeler ainsi les pièces dont l'étendue permet un spectacle entier) sont devenues souvent des "pièces fleuves [...] constituées d'une accumulation de formes brèves, d'une série de fragments ${ }^{7}$ ». Tel était déjà l'aspect que présentait Tristan et Yseult, mais la pièce demeurait centrée sur l'aventure tragique des deux personnages éponymes, alors que le texte de Daniel Danis réinscrit l'histoire - vécue ici de manière communautaire - , dans une dynamique plutôt épique. Aussi doit-on prendre acte de la présence de ce personnage qu'est la Didascalienne, qui ouvre d'entrée de jeu le récit par ces paroles simples: «Dis ce que vois.» (13) D'une certaine manière, la pièce raconte son histoire à elle, c'est-à-dire la manière dont elle est devenue ce témoin privilégié, qui dit ce qu'elle voit ou plutôt ce qu'elle $a v u$, mais également la manière dont elle a été autorisée à livrer ce témoignage. Il faut que le verbe dire se conjugue au verbe voir pour qu'il y ait témoignage.

En effet, e raconte l'histoire d'un personnage nommé J'il - lui manquerait-il aussi le «e»? - depuis sa naissance, sous les bombes, jusqu'à sa mort, au moment où il allait négocier l'armistice. Tel Oreste, J'il porte les souffrances de son peuple, réfugié dans un Autre Monde qui ne veut pas de lui: «Ils ne sont ni un peuple ni une race; personne ne veut de cette racaille qui colle sur les terres qu'ils squattent." (21) Commentant Le chant du Dire-Dire, Yves Jubinville signalait déjà, chez Danis, l'existence $d^{\prime}$ ' un imaginaire des premiers temps, donnant accès à un mode archaïque de perceptions et de socialisation ${ }^{8}$ ", imaginaire que l'on retrouve ici dans ces circonstances similaires. L'histoire de J'il peut donc être lue comme celle d'un individu qui parvient à dépasser le mode archaïque de perceptions que lui imposent les circonstances - mode fondé sur la violence de la guerre, qui engendre le meurtre, et encore la guerre - , et qui parvient à saisir la nécessité de déployer un nouveau mode de socialisation: «Il ne faut plus se venger.» (117) Lui, qui a appris à lire en prison en écoutant à travers les murs les leçons données aux autres prisonniers, rencontre Romane, une fille de l'Autre Monde, qui, après avoir accouché de deux jumeaux baptisés Jadis et Demain, constitue un registre des réfugiés du camp et, de cette manière, fait entrer la communauté dans le régime du droit. La transformation ne va pas de soi et la tragédie les rattrape quand J'il découvre que Romane était sa sœur et que le peuple, soulevé par ses passions, se débarrasse de cette famille encombrante. Mais, à terme, la volonté de Romane de donner la vie et d'écrire la mémoire du monde aura peut-être raison de la violence. Car c'est sa fille Soleil, échappée du massacre et devenue la Didascalienne, qui reprend le fil du récit pour assurer la continuité: «Ainsi, moi, Soleil, ai la tâche d'écrire qu'avec ces mots s'achève le roman-dit de J'il.» (125)

7 Jean-Pierre Sarrazac, «Réflexion sur un moment d'histoire de la forme courte au théâtre», Les Cahiers de Prospero, n 10, 2000, p. 103. 8 Yves Jubinville, «La vie en reste. Sur quelques cas de témoignages dans la dramaturgie québécoise actuelle (Danis, Chaurette, Tremblay)», Chantal Hébert et Irène Perelli-Contos (dir.), op. cit., p. 51. 
La mémoire du monde se fixe parfois autrement que par le récit. La dernière pièce de Michel Marc Bouchard, Le peintre des madones ou La naissance d'un tableau ${ }^{9}$, que l'auteur qualifie de "conte», raconte la genèse de la fresque de Saint-Cœur-deMarie. Ce sont les habitants qui auraient inspiré "cette histoire avec leurs chuchotements sur les querelles conjugales entre Dieu et Satan» (7). Créée à Florence, au Teatro della Limonaia de Sesto Fiorentino, en juin 2002, la pièce a obtenu le Primo Arte Candoni 2002 pour la meilleure nouvelle pièce en langue étrangère jouée en Italie. Créée dans une version florentine au même endroit en novembre 2003, puis reprise à Rome, elle a été présentée en français à Montréal à l'Espace Go, en avril 2004, dans une mise en scène de Serge Denoncourt, assisté de Ginette Lagacé. On comprend que les circonstances particulières de cette création italienne aient pu suggérer à l'auteur le choix du motif artistique et ce personnage de "peintre italien qui vagabonde en nos terres [et qui] a l'originalité de prendre à chaque fois comme modèle une jeune fille de l'endroit» (28). La pièce assure ainsi la rencontre de deux univers historiques profondément marqués par une puissante tradition religieuse: l'italienne, que représente ce peintre vagabond, et la québécoise, portée par un jeune prêtre qui désire "enorgueillir [son] église d'une grande fresque dédiée au cœur sacré de la Vierge Marie» (27). Toutefois, le fait que cette rencontre se produise à propos d'une œuvre d'art n'est pas sans soulever quelque passion, que va encore exacerber le temps de l'action, qui se passe à la fin de 1918, au moment où les soldats ramènent du front le terrible virus de la grippe espagnole. L'œuvre d'art n'est-elle pas une manière de conjurer le temps et de défier la mort?

Découpée en 18 tableaux, la pièce est encadrée d'un prologue et d'un épilogue pris en charge par l'Ange annonciateur: «Et arrivera le porteur de tous les espoirs; celui par qui tout un chacun se révèlera comme il se doit d'être révélé. » (39) L'enjeu de l'action dramatique est de trouver un modèle pour le visage de la Vierge de Marie. Entrent alors en concurrence les jeunes filles du village: Marie-Paule, Marie-Anne et Marie-Louise, à qui l'artiste préférera d'abord Marie des Morts, vaguement sorcière, qui a le don de faire mourir les malades. Cependant, dans un village sans hommes - les soldats sont encore au front, les déserteurs sont dans le bois -, il est à prévoir que les choses se détraquent. Ainsi, pour se venger et après l'avoir embrassé, Marie-Paule jette le cadavre d'un soldat anglais mort de la grippe dans la rivière, contaminant l'eau du village. Marie-Anne, qui hante les bois à la recherche d'un hypothétique fiancé, finit par séduire le curé, dont le médecin disait déjà qu'il était «trop beau pour un prêtre» (23). Marie-Louise, qui lit dans les draps, est dévastée de découvrir la passion qui unit le peintre à Marie des Morts, laquelle, ayant découvert l'amour, perd progressivement ses pouvoirs. Pourtant, cette passion se révèle insuffisante à produire l'œuvre d'art recherchée. Finalement, expiant son péché à lui, mais aussi tous les péchés du monde, le curé se soumet au scalpel du

$++$

9 Michel Marc Bouchard, Le peintre des madones ou La naissance d'un tableau, Montréal, Leméac, coll. «Thêâtre», 2004, 99 p. 
docteur qui livrera son visage au peintre. La réalisation de la fresque agit ainsi tel un exorcisme, où se seraient affrontés l'art et le sacré, le peintre et le prêtre, le diable et le bon dieu (à moins que ce ne soit l'inverse). Que l'exorcisme se produise par l'inversion des genres sexués, le prêtre prenant la place de la sorcière, n'est pas sans rappeler d'autres textes de l'auteur, notamment Les feluettes, dont Le peintre des madones reprend la structure en trompe-l'œil, s'autorisant cette fois de la mémoire collective, plutôt que de celle d'un personnage unique, pour révéler les crimes (ou les péchés) sur lesquels s'est construit le présent: «Et, de conteurs en conteurs, d'oblats en diacres, ils rendirent l'histoire encore plus diabolique qu'elle ne l'était vraiment. Ce fut leur façon de défier la peur de la mort. En vérité, en vérité, je vous le dis.» (99)

Dans quelques années, qui se rappellera encore la comédienne formidable que fut Gisèle Schmidt? Julie Vincent, qui avait déjà exploré la figure de l'actrice dans Noir de monde (1989), y revient dans La robe de mariée de Gisèle Schmidt ${ }^{10}$, pièce créée à l'Espace Go, le 21 mars 2006, dans une mise en scène de l'auteure. Il ne s'agit pas ici d'une biographie, ni même d'une réflexion sur le métier de l'actrice ou sur la femme réelle qui se cache derrière la professionnelle. Nous aurons plutôt six textes brefs (synthèse dramatique, scène unique, sketch, comme on voudra), qui se déroulent à six moments et six lieux distincts. L'auteur les a nommés "nouvelles» plutôt que "pièces» - qu'importe! les genres brefs ne sont pas si éloignés les uns des autres -, et leur réunion en un cycle unique emprunte à l'esthétique du recueil la nécessité de créer son unité, aussi ténue qu'elle soit. Ainsi, le personnage de Gisèle Schmidt paraît quelques instants chaque fois dans les «entre-scènes » qui assurent la transition d'une nouvelle à l'autre. Les quelques phrases qu'elle prononce sont tirées des six entretiens que Julie Vincent a eus avec elle à la fin de sa vie, comme en un hommage que présenterait une actrice à une autre, plus âgée. Il rappelle le thêâtre, le temps qui passe, parfois rien du tout. Elle est évoquée encore par la présence constante sur la scène d'un fauteuil et de quelques objets, un fume-cigarette, une cape rouge, des mains. Quant à la robe de mariée, elle est un objet de fiction, l'actrice n'en ayant jamais eue. L'auteur la conçoit comme « un moyen de transport » : " [T]irée de la rêverie amoureuse de Gisèle, une robe de mariée voyage d'un pays à l'autre et se transforme six fois sur une période d'à peu près trente ans.» (14)

Transportés, nous le sommes, à travers ces anecdotes diversement inspirées de Louis Aragon, d'Anton Tchekhov, d'Oscar Wilde et des actualités. Dans le métro de Montréal, en juillet 2003, deux comédiens (elle en robe de mariée) se caressent sous les yeux amusés d'une vieille dame. Dans une usine de textiles, en octobre 1970, deux immigrants, elle Portugaise et lui Yougoslave ont une aventure, à l'heure de la pause (elle revêt alors la robe de mariée). Au Pirée, en 1975, Simone a caché des

10 Julie Vincent, La robe de mariée de Gisèle Schmidt. Nouvelles-théâtre, Montréal, Les Éditions de la Pleine lune, 2004, 93 p. 
billets de banque et du haschich dans les ourlets de sa robe de mariée. À Nîmes, en 1981, un guitariste manchot (!) offre à une prostituée une robe de mariée volée à Athènes et lui propose de partir au Canada. À Chicoutimi, en 1988, pendant le Carnaval du bout du monde, une docteure en physique nucleaire (nous sommes quelques mois après les événements de Tchernobyl) revêt une robe de mariée comme costume. Dans un lieu et un temps imprécis, le conseiller financier d'une caisse populaire dépose une robe de mariée dans le cercueil de l'homme qu'il aimait, mort du sida. L'ordre, on le voit, n'est pas chronologique, ni l'espace strictement linéaire. Ces nouvelles "en forme de thêâtre» présentent ainsi des moments dramatiques intenses, dont on ne connaît ni l'origine ni la fin et, à vrai dire, pas même le déroulement. Dans le prologue qui les précède, l'actrice qui personnifie l'auteure explique les avoir écrites "pour défier le temps» (14). Suspendre le temps en lui refusant toute linéarité, le figer à une date précise, dont le passé et l'avenir nous échappent, apparaît dès lors comme une manière d'atteindre l'éternité.

«Les acteurs sont des passeurs de rêve» (14), disait Gisèle Schmidt à Julie Vincent. Les metteurs en scène seraient, pour leur part, des Passeurs d'âmes ${ }^{11}$, selon Martine Beaulne, comédienne et metteure en scène, qui publie ici son premier essai. L'événement mérite d'être souligné, car le fait qu'un metteur en scène prenne la plume pour écrire est rare. La plupart du temps, il faut attendre la bonne volonté d'un journaliste ou d'un chercheur qui, avec son magnétophone, recueillera les propos de l'un ou de l'autre. Même dans ce cas, il faut bien le dire, le corpus reste bien mince et, à bien y réfléchir, il faut remonter au début du vingtième siècle pour retrouver le genre d'essai que propose ici Martine Beaulne, dans Comédiens et amateurs (1919) d'Eugène Lassalle, fondateur du conservatoire qui a longtemps porté son nom. Et, comme s'il y avait là une manière commune de concevoir la relation à l'écriture, Beaulne construit son essai de manière assez semblable. Le propos est plus moderne, bien sûr, le caractère autobiographique plus intime, la description des mises en scène réalisées plus précise, le projet esthétique plus développé. Nous y gagnons.

Troisième d'une longue lignée de metteurs en scène (c'est en 1905 que Léonard Beaulne fonde à Hull sa première troupe, le Cercle Crémazie), Martine Beaulne témoigne de cet héritage de pionniers et rend hommage à son père Guy, qui lui a transmis sa passion du thêâtre en même temps qu'une certaine rigueur dans la conception du spectacle. L'on sent néanmoins, dans cette trajectoire qu'elle revit, l'inquiétude qu'elle a pu éprouver à assumer l'héritage, puis à assurer la relève. Directrice artistique des Treize de l'Université Laval (1971), membre du Thêâtre Parminou (1974-1983), l'auteur a pris quinze ans pour affirmer son identité propre et devenir, à son tour, un passeur d'âmes : «En devenant un passeur d'âmes à travers la pratique de la mise en scène, j'ai cru que je pouvais mettre en lumière les mots,

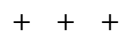

11 Martine Beaulne, Le passeur d'âmes. Genèse et métaphysique d'une écriture scénique, Montréal, Leméac, coll. «L'écritoire», 2004, 197 p. 
faire entendre la musique d'une humanité et mettre en regard un univers symbolique. » (17) Telle est en effet la conception que Martine Beaulne se fait de la mise en scène, dont elle écrit encore qu'elle sert à favoriser les rencontres entre un auteur et un acteur, entre un personnage et un spectateur. Passer, c'est ainsi transmettre. Toutefois, le passage n'est jamais pure transparence; il engage un travail neuf, à la fois une construction (du spectacle) et une création (d'un sens nouveau). Elle revient alors sur quelques-unes de ses mises en scène, sans doute les plus significatives à ses yeux : L'arbre des tropiques de Yukio Mishima (La Veillée, 1990), Don Juan de Milosz (La Veillée, 1991) et de Molière (TNM, 2000), La Locandiera de Carlo Goldoni (TNM, 1993), Albertine en cinq temps de Michel Tremblay (Espace Go, 1995). Elle relit ses mises en scène et les réinvestit, en même temps qu'elle fixe dans notre mémoire ses principes et ses choix esthétiques. Au terme de ce parcours, qui a ainsi remonté le fil de la mémoire, elle peut enfin conclure, réconciliée avec elle-même:

Le passeur d'âmes est un voyage intérieur qui a tenté de communiquer les rencontres vécues avec des existences visibles et invisibles, de faire entendre les voix connues et inconnues qui ont hanté mon univers intérieur. Ces âmes qui ont envahi l'espace fictif de la scène m’ont émue, provoquée. J'ai pu déterminer les sources affectives des différentes symboliques présentes dans mon écriture, analyse qui m’a réconciliée avec moi-même et avec certains événements de mon histoire personnelle et de notre histoire collective. (182) 